\title{
PARQUES NATURALES ANDALUCES: UNA ESTRATEGIA DE CONSERVACION Y DESARROLLO EN REGIONES DEPRIMIDAS
}

Fernando DIAZ del OLMO *

Fernando MOLINA VAZQUEZ **

Cuando en 1980 publican la UICN el PNUMA y la WWF la Estrategia Mundial para la Conservación, se ha consolidado en los países occidentales la idea del proteccionismo de la naturaleza, culminándose de esta manera un proceso que claramente arranca de los prolegómenos de la Conferencia MAR (1962), Ramsar (1971), Estrasburgo (1970), Estocolmo (1972), pasando por el Programa MAB de la UNESCO. Puede decirse que ha emergido una nueva interpretación de la sociedad, de corte conservacionista, no exenta de tensiones.

Obviamente el campo de operaciones de las políticas proteccionista va a encontrar mayores posibilidades en aquellos conjuntos geográficos donde a la fecha existía un menor esquilmamiento de las condiciones naturales primitivas. No es casualidad que exista una constante relación entre áreas conservadas y regiones subdesarrolladas. Andalucía es un inmejorable ejemplo de economía de subdesarrollo (CANO, 1983; DELGADO CABEZA, 1984, entre muchos), habiéndose propuesto en diferentes ocasiones vías de desarrollo al amparo de políticas económicas (CAPELO, 1963; PAYNO, 1983).

En lo que sigue exponemos cómo la idea de protección, bajo el prisma de la figura de Parque Natural, sirve de modelo integral de un ecodesarrollo.

\section{LA DEFORESTACION COMO HECHO SELECTIVO PARA LA CONSERVACION}

El modelo de ocupación del territorio andaluz dista mucho de ser homogéneo habida cuenta de la variabilidad geográfica y ecológica que presenta la Comunidad Autónoma. Sin embargo, a partir del conocimiento de los actuales paisajes vegetales, sucesiones fitosociológicas, y las referencias históricas

* Profesor de Geografía. Universidad de Sevilla.

* Biólogo, Agencia de Medio Ambiente. Junta de Andalucía. 
de los aprovechamientos agrícolas y ganaderos, es posible sintetizar la dinámica que a grandes rasgos ha conducido a la transformación actual. Minimizando, se puede hacer un esquema de la deforestación que parte de un aclareo de bosques según se iban poblando valles y montañas. En primer lugar la desaparición actúa en las cotas más bajas, de topografía más llana y situación favorable, como son las áreas litorales, vegas, terrazas fluviales, y en general terrenos no inundables. Los bosques, maquis y garrigas se relegaban a las áreas montañosas con topografía abrupta y suelos pobres con dificultades para su transformación agrícola. En éstas las condiciones económicas se basaron en la cabañas ganaderas que al tener un fuerte carácter extensivo no permitió un desarrollo comparable con las áreas agrícolas. La falta de una eficaz articulación territorial de los conjuntos serranos con el resto de las comarcas, completa la idea de aislamiento.

De esta manera encontramos en las áreas montañosas los parajes mejor conservados al funcionar de manera selectiva respecto del medio físico los procesos de desforestación, con bosques de aestilignosas (robledales, quejigales), durilignosas (algarrobos, encinares), y aciculilignosas (pinsapares, pinares), combinándose en mayor o menor medida con las formaciones subarbustivas. Los sistemas de explotación, hoy como ayer, siguen de cara al monte, primando la ganadería y sus productos derivados, complementándose a base de una agricultura tradicional y actividades marginales (caza, apicultura, carboneo, recolección).

La situación parace invertirse en los momentos actuales, expoleada por las circunstancias críticas tanto de tipo demográfico como de tipo económico, pasándose a una ocupación intensiva del monte, como secuela de un uso intensivo no programado: repoblaciones irracionales, urbanizaciones incontroladas, pastoreo abusivo, alto furtivismo cinegético e incendios y talas de grandes extensiones. Estamos refiriéndonos a los casos conocidos de las serranías de Grazalema y Montes de Algeciras, en la provincia de Cádiz, Sierra Nevada en Granada y Hornachuelos en la Sierra Morena cordobesa, como ejemplos suficientemente representativos.

\section{CIENCIA Y ESTRATEGIA EN LA CONSERVACION DE ANDALUCIA: ESPACIOS NATURALES Y PARQUES NATURALES}

Para poder contrarrestar los impactos ambientales, la política protecciomista pasa por ser ordenada e integral. Con este ánimo se ha emprendido en nuestra región la conformación de una red básica de interés naturalístico con la pretensión de ir dando las figuras jurídicas oportunas, desde Reservas a modalidades inferiores, a los múltiples espacios de interés. Así pues trata de alcanzarse dos fines que aunque coaligados tienen una cierta autonomía: 
a) Corformar la Red de Espacios Naturales Andaluces, inspirada en las Reservas de la Biosfera (CASTRI Y LOOPE, 1977), y por lo tanto desarrollando a nivel regional los criterios de representatividad, diversidad, carácter natural y eficacia como unidades de conservación referidas a los ecosistemas de los lugares a proteger.

b) Catalogar las provincias una a una (JUNTA DE ANDALUCIA, 1981).

Un concepto tal como espacio natural a una demarcación geográfica con protección. Pero, ¿Cual es su valor?. En sí mismo tiene una fuerte carga bivalente, relativo tanto a los caracteres geoecológicos estrictos como a la capacidad de acción antrópica sobre él, lo cual provoca al menos tres lecturas o interpretaciones diferentes:

- De conservación: Directamente asociada a la escasez de recursos naturalísticos cuya máxima expresión quizá sea el documento EMC (Estragegia Mundial para la Conservación). Conservación es "La forma de utilización de la Biosfera por el ser humano de tal suerte que se produzca el mayor y sostenido beneficio para las generaciones actuales, pero manteniendo su potencialidad para satisfacer las necesidades y aspiraciones de las generaciones futuras" (ABOAL, 1982). A resaltar pues que conservación no quiere decir eliminación de aprovechamientos sino racional utilización de los recursos naturales. Atajaría de esta manera la expoliación de los ámbitos selectivizados por la desforestación anteriormente aludida.

- Educacional: Afectando decididamente a los niveles pedagógicos infantiles y juveniles pasando por la implementación de albergues, itinerarios, visitas y monitores, completándose con la idea de promoción social y cultural de los municipios implicados.

- De esparcimiento: Configurando una de las alternativas claves para la coordinación de estos espacios con el resto del territorio, planificándose sus necesidades urbanísticas por medio de las medidas cautelares que se expresan en los Planes del Medio Físico (MARCHENA, 1984; ACOSTA, 1984; EPYPSA, 1984; a y b; VERDE 1984; CAGGIANO Y SANZ, 1984).

La no armonización de esta trilogía conlleva una alta carga de recesión que ataca directamente a la médula de cualquier programa de actuación medioambiental sobre el territorio. Prueba de ello fue el descrédito de nuestra Ley de Espacios Naturales Protegidos de Mayo de 1975. 
En este orden de cosas surge con grandes posibilidades la categoría de Parque Natural. Su filosofía acoge proteccion y recreación (ANGLADA, 1983). Se aplican a unidades ambientales de orden polimunicipal. No cercena ni las explotaciones forestales ni las agrícolas-ganaderas o las cinegéticas. Atiende al desarrollo de los productos tradicionales y al mejor aprovechamiento de la potencialidad del medio, combinándose la posibilidad de establecer Reservas Integrales o protecciones especiales dentro de su marco de actuación. En nuestro país esta figura está aún comenzando a desarrollarse, cuando no en ciernes: en la Comunidad Vasca se han perfilado 16 Parques Naturales (RUIZ DE URRESTARAZU, 1983); por la Diputación de Barcelona y a través de Planes Especiales por la Ley del Suelo, se ha declarado cuatro Parques Naturales (DIPUTACION DE BARCELONA, 1984); en esta misma idea está la reciente aprobación del Parque Regional del Manzanares en la Comunidad Autónoma de Madrid (Enero, 1985). En Francia la Red de Parques Naturales tiene una amplia implantación, afectando a 18 Regiones, 39 Departamentos, 1.300 Comunas y totalizando su acción sobre 860.000 habitantes (LES PARCS NATURELS... 1982).

Sin embargo esta figura ha originado en Andalucía controversias infundadas fruto del confusionismo con la de Parque Nacional, entre los habitantes de las distintas áreas rurales. Los objetivos de ambos Parques son diferentes. En el Nacional se atiende a la preservación, es decir, a mantener el estado natural de un área o de una categoría de seres vivientes, poniéndola a cubierto de daños (por ejemplo, el Parque Nacional de Doñana, por lo demás el único existente en Andalucía). Los aprovechamientos pasan a un segundo o tercer plano, pudiendo incluso ser anulados cuando interfieran con la preservación del espacio. El uso del Parque para el acercamiento del hombre a la naturaleza queda también relegado, aunque sea uno de los fines de dicha figura jurídica, cuando no sea compatible este acercamiento con la conservación.

\section{LOS PARQUES NATURALES ANDALUCES}

Al amparo de la Ley de Espacios Naturales Protegidos de Mayo de 1975, ya citada, Andalucía cuenta con dos Parques Naturales declarados: el del Torcal de Antequera, Málaga, con 1.200 Ha. y el de Sierra de Grazalema, Cádiz-Málaga, con 47.000 Ha.

Otros Parques se preveen su declaración. De su importancia territorial puede dar una idea el cuadro I. De todos ellos el de mayor dimensión y quizá el más ambicioso corresponda al de Sierra Nevada, dada la multiplicidad de usos y caracteres que en él se concitan. En la provincia de Cádiz los Parques del Aljibe y Montes de Algeciras van a tratar de potenciar estas Sierras con excelentes nuasas de alcornocar, históricamente desarticuladas entre 
el Campo de Gibraltar y las Campiñas gaditanas. Al norte del Valle del Guadalquivir, entre Sevilla y Córdoba el área de Hornachuelos reune los caracteres naturalísticos y comarcales precisos para su declaración como Parque $\mathrm{Na}$ tural, con formaciones de encinar y matorral y usos múltiples de adehesados. La Sierra de las Nieves en la provincia de Málaga, entre la Costa del Sol y Ronda, con su riqueza biológica, ha conformado tradicionalmente un espacio marginal que debe ser utilizado y conservado dentro de las posibilidades regionales. Finalmente el área de Cazorla, muy conocida y visitada debe de regularse de cara a controlar los negativos impactos ambientales. En el mapa n.o 1 se recoge la distribución de estos espacios que a nuestro entender debe componer la Red básica de Parques Naturales Andaluces.

Acerca de la declaración del Torcal de Antequera como Parque Natural se suscitó una viva polémica sobre su reclasificación a Paraje Natural de Interés Nacional, zanjada a finales del pasado año 84 por el Parlamento andaluz a favor de la primera denominación.

\section{CUADRO I}

RED BASICA DE PARQUES NATURALES. FEBRERO 1985

\begin{tabular}{|c|c|c|c|c|c|}
\hline & Parque & Ha. & $\begin{array}{c}\text { Municipios } \\
\text { afectados } \\
\left(n .^{\circ}\right)\end{array}$ & Provincia & $\begin{array}{l}\text { Estado } \\
\text { actual }\end{array}$ \\
\hline 1. & Torcal de Antequera ..... & 1.200 & 1 & Málaga & Declarado \\
\hline 2 , & Sierra de Grazalema. ...... & 47.000 & 13 & Cádiz-Málaga & Declarado \\
\hline 3. & Sierra de Aljibe ........ & 100.000 & 8 & Cádiz-Málaga & En estudio \\
\hline 4. & Montes de Algeciras. ..... & 40.850 & 4 & Cádiz & En estudio \\
\hline 5. & Hornachuelos ........ & 70.000 & 5 & Córdoba-Sevilla & En estudio \\
\hline 6. & Sierra Nevada . . . . . . . & 225.000 & 72 & Granada-Almería & En declaración \\
\hline 7. & Sierra de las Nieves ...... & 14.125 & 4 & Málaga & En declaración \\
\hline 8. & Sierra de Cazorla y Segura ... & 177.873 & 22 & Jaén & En declaración \\
\hline
\end{tabular}

El Parque del Torcal está determinado naturalísticamente por un prioritario interés de corte geológico y geomorfológico, donde a un singular aparato kárstico se le añaden manifestaciones de signo periglaciar, con una compleja evolución que ha cristalizado igualmente en la aparición de endemismos botánicos que enriquecen florísticamente la geografía de las Béticas. Por ello, a la valoración como Parque o Paraje Natural creemos debería añadírsele otra que con mayor precisión se ajustase a los peculiares rasgos anteriormente sintetizados; nos referimos a la apuntada por ANGLADA, 1983, de Monumento Natural, "elemento singular de la naturaleza de reducida extensión cuya protección responde a un interés científico o cultural", figura ésta de preciado valor para la futura Ley de Protección de la Naturaleza, ya que daría cabida, de esta manera, a los conjuntos físicos de peculiaridades concretas. 


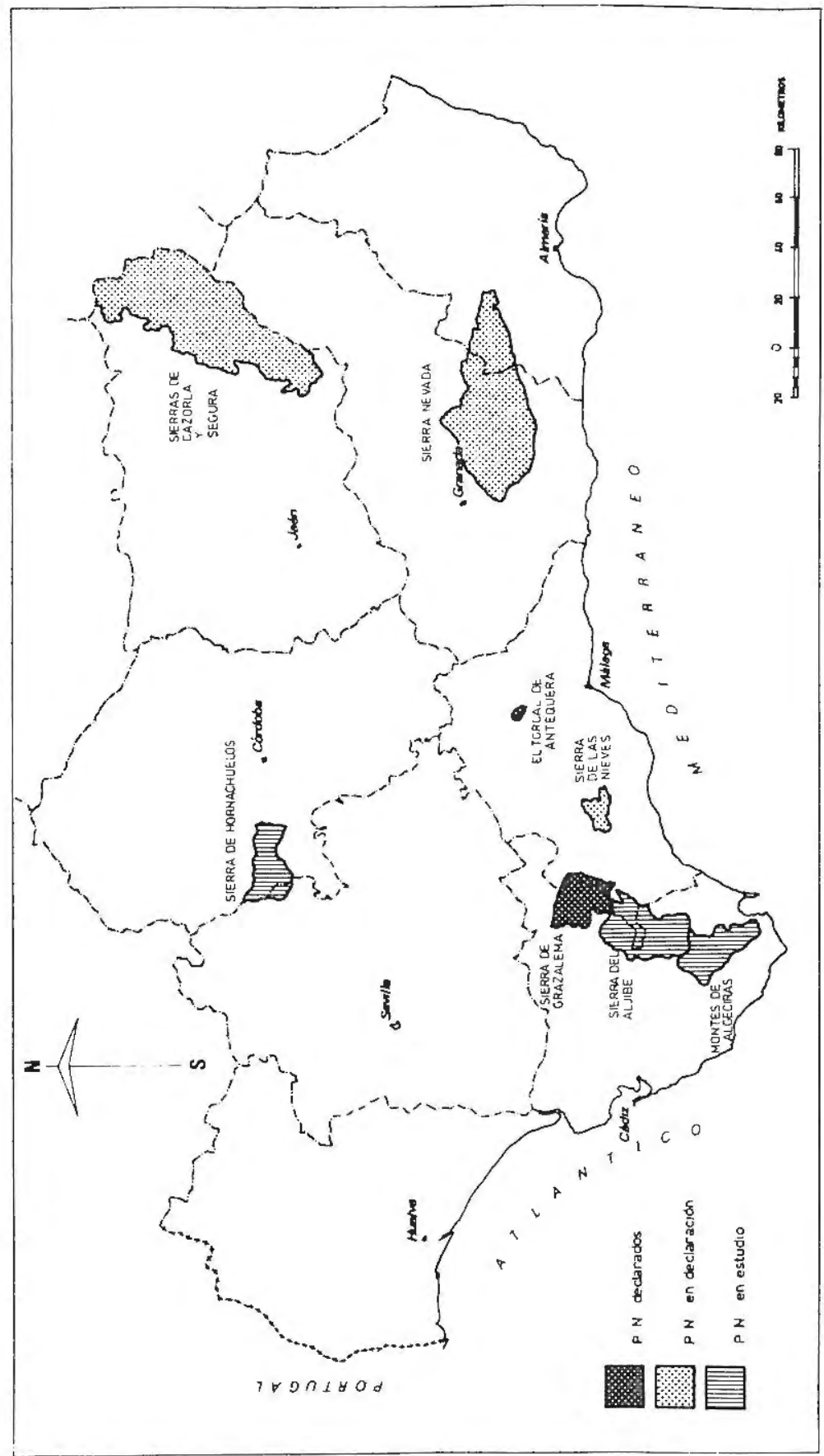


En este sentido la figura de Parque Natural se ajustaría a aquellas áreas de gran extensión con palpable necesidad de ordenación y conservación de sus recursos naturales. Mención importante es el caso del Parque Natural del Macizo del Garraf (Barcelona), con una fuerte recesión rural y una aguda presión como espacio de ocio prolongación del núcleo urbano de Barcelona. El Plan Especial elaborado para la declaración del Parque tiene como finalidad primordial la restauración del paisaje y promoción de una función social ordenada.

El interés físico-natural del P.N. de Grazalema, mudialmente reconocido como se demuestra en la demarcación de Reserva de la Biosfera que planteara la UNESCO en 1978 para la conservación de sus sector central, se basa en un conjunto de rasgos que abarcan variantes geológicas, geomorfológicas, espeleológicas, botánicas, ecológicas y zoológicas. Su demarcación se extiende por las sierras del Pinar, Endrinal, Caillo, Margarita, Ubrique, Líbar, Zafalgar y Labradillo, totalizando más de $40.000 \mathrm{Ha}$., entre las provincias de Cádiz y Málaga, formando parte de las unidades geológicas de las Béticas Externas. El núcleo más resaltable corresponde al eje Pinar-Endrinal, separados por el corredor del Boyar, en las inmediaciones del pueblo de Grazalema, donde se alcanzan las altitudes máximas de Cádiz (1654 metros), y el régimen pluviométrico más elevado de la península (por encima de los $2.000 \mathrm{~mm} / \mathrm{año}$ ). Estas condiciones han conformado formaciones biogeográficas mixtas y peculiares, con encinares, alcornocales, quejigares y pinsapares, estos últimos resto de una vegetación endémica y relicta, individualizada durante las crisis climáticas pleistocénicas.

Completando lo anterior existe todo un patrimonio cultural de honda raigambre histórica con manifiestas singularidades desde el XVIII, y centro en Grazalema y Ubrique. Desde entonces aquí se han mantenido las actividades artesanales y rurales al tiempo que las formas arquitectónicas, cebándose en toda la serranía la marginación típica de las áreas de montaña del mundo mediterráneo.

En Diciembre de 1984 la Agencia de Medio Ambiente de la Junta de Andalucía declara al Macizo de Grazalema como Parque Natural, desplegando previamente una serie de actividades informativas (Exposierra '84, HURTADO, 1984). Se aborda así y de manera acertada, la necesidad de protección de este entorno no olvidándose de su revitalización, ya que de forma paralela se lleva a cabo un Plan de Actuación conjunto con otros organismos públicos de la administración local, provincial y autonómica. Consiste en la potenciación de los sectores ganaderos, por medio de la ordenación de montes y transformación racional de las cabañas; textiles, recuperando las actividades artesanales; chacineros, apoyando un mejor sistema comercial; turístico, con una mejora de la infraestructura de visita, estancias y esparcimiento; piscícola, revalorizando los Cotos de Pesca y las piscifactorias existentes; y 
artesanales, que encauza directamente toda la explotación de los amplios recursos naturales (apícolas, queseros, cinegéticos). En resumen, y según venimos insistiendo a lo largo del artículo, pretende compatibilizar la protección de los valores y recursos naturales con el desarrollo racional del área, realzando así el binomio conservación-desarrollo. Todo esto no significa más que un primer acercamiento a los necesarios Planes Rectores de Uso y Gestión (PRUG), que obviamente deben acompañar a la declaración de Parques Naturales.

El caso de Sierra Nevada supone un verdadero reto de puesta a punto y banco de pruebas del potencial territorial que encierra la figura de Parque Natural. Según el estudio previo realizado por el ICONA (DE BENITO y col., 1984) englobaría las comarcas de la Sierra Norte, Marquesado de Zenete, Valle del Lecrín y parte de la Alpujarra, en Granada, y Valle del Río Nacimiento y Andarax, en Almería, en proporciones variadas. La posición geográfica de este núcleo orográfico conlleva un nítido escalonamiento bio y morfoclimático con un interés excepcional en nuestra región. Al mismo tiempo la existencia de la estación de montaña con promoción turística enlazada con las comarcas del entorno, ha introducido una componente socioeconómica distinta a los usos tradicionales que se dan en los demás Parques.

La diferencia del ámbito de las Sierras de Cazorla y Segura (Jaen) respecto de otros espacios naturales estriba tal vez no tanto en los caracteres geológicos o biológicos (excepción hecha de sus endemismos), como en el másivo uso de campismo y cinegético, explotado de forma prioritaria por un turismo procedente de la submeseta meridional. De esta manera una futura delimitación de Parque Natural debe apoyar la ordenación de visitas, educación ambiental y reorganización de las infraestructuras comarcales así como una revisión de las actividades cinegéticas en relación con las especies introducidas en el marco de lo que hoy es Reserva Nacional de Caza. Igualmente esta revisión cinegética debe aplicarse a la Reserva Nacional de Caza de Sierra de las Nieves (Málaga), estando parte de ella actualmene en estudio para su declaración como Parque Natural, atendiendo a la articulación de toda la geografía de la serranía de Ronda.

Finalizando la Red Básica de Parques Naturales Andaluces, están tres grandes áreas. Al norte del Campo de Gibraltar se encuentran las Sierras que componen los llamados Montes de Algeciras y las sierras del Aljibe. Su delimitación puede presentar dificultades habida cuenta de su homogeneidad geológica, geomorfológica y biogeográfica: series flyschoides silicícolas fuertemente tectonizadas, con un potente alcornocal acompañándose en los barrancos por relictos de laurisilva. No obstante el uso histórico de las masas forestales supone una diferenciación paisajística, al tiempo que un posible criterio, entre las sierras meridionales y las septentrionales. A resaltar esta el hecho de que Cádiz es la provincia de mayor extensión de alcornocal de España, centrándose en estas comarcas las más de 80.000 Ha. de Quercus suber, 
por las posibilidades que en un PRUG tendrían los proyectos de aprovechamientos industriales corcheros. En el sector central de Sierra Morena, entre Sevilla y Córdoba, se ubica la zona de Hornachuelos, comarca eminentemente rural, con todos los ingredientes típicos del entorno mariánico: marginación económica, adehesados, minero y cinegéticos.

\section{CONCLUSIONES}

De lo que se ha expuesto hasta aquí se pueden deducir varias cuestiones:

1) Sobre la figura de Parque Natural. Trata de perfilar una política con una intensa filosofía de recreación, esparcimiento y potencialidad de los recursos naturales, enlazando a los municipios afectados.

2) Sobre los criterios de delimitación de un Parque Natural. Tres contenidos esenciales lo perfilan: el polimunicipal de gran extensión (orden de magnitud de miles de $\mathrm{Ha}$ ); la homogeneidad fisico-naturalística; y la cultural y etnológica.

3) Sobre la problemática de designación de Parque Natural. Quizá sea este un punto de delicado tratamiento habida cuenta de las reticencias existentes en los ámbitos rurales por entrar a formar parte de entidades territoriales mayores, basado en definitiva en una desacertada política informativa acaecida en las planificaciones de nuestra historia inmediata, en donde no tenía cabida la opinión del habitante rural.

\section{BIBLIOGRAFIA CITADA}

ABOAL, J. L., AZCARATE, T. (1982). "Seminario sobre recursos naturales: bosques, zonas húmedas y zonas árides semiáridas". Ponencia. Madrid (inédito).

Acosta BONO, G. (1984). "Los Planes Especiales de Protección del Medio Físico". Revista de Estudios Andaluces, n. ${ }^{\circ} 3$, pp. 161-176.

ANGLADA GOTOR, S. (1983). "Criterios para una ley básica de protección de la naturaleza: régimen de los espacios naturales protegibles”. Informe elaborado para el MOPU. (Inédito).

ARISO, A. (1982). "La actitud de las comunidades ruraies ante la protección de los espacios naturaies". I Encuentro de Expertos sobre la gestión de los Parques Naturales y Reservas Equivalentes. Diputación de Barcelona. pp. 8-10.

GAGGIANO, R. y SANZ, E. (1984). "Los PEPMF y las evaluaciones de impacto ambiental dentro del proceso de planificación socioterritorial". Primeras Jornadas de Impacto Ambiental. Granada.

CANO GARCIA, G. (1983). "Unidad y diversidad de la geograffa andaiuza". Revista de Estudios Andaluces, $\mathrm{n} .{ }^{\circ} 1, \mathrm{pp}, 9-22$. 
CAPElo martinez, M. (1963). "Fundamentos del desarrollo económico de Andalucia". C.S.I.C. Madrid.

CASTRI, F, y LOOPE, LL. (1977). "Reservas de la Biosfera: teoría y práctica". La Naturaleza y sus recursos. Vol. 13, n. ${ }^{\circ} 1$ pp. 2-8.

DE BENITO, J. M.; IR URITA, J.; ROMERO, M. (1984). "Estudio previo a la declaración del Parque Natural de Sierra Nevada". ICONA. Novena Ins. Reg. (Inédito).

DE BENITO, J. M.; MOLINA, F. (1983). "Estudio previo para la declaración de la Sierra del Aljibe". ICONA. Décina Ins. Reg. (Inédito).

DELGADO CABEZA, M. (1984). "Reflexiones sobre algunos obstáculos paxa aproximarnos a la realidad económica de Andalucía”. Revista de Estudios Andaluces, n. ${ }^{\circ}$ 3, pp. 15-32.

DIPUTACION DE BARCELONA. (1984). Servei de Parcs Naturals. Documentos internos. (Inéditos).

EPYPSA (1984). "Plan Especial de Protección del Medio Físico y Catálogo de Espacios y Bienes Protegibles de la provincia de Cádiz". Documento y Síntesis. Consejería de Política Territorial. J. A. Sevilla.

EPYPSA (1984). "Plan Especial de Protección del Medio Físico y Catálogo de Espacios y Bienes Protegibles de la provincia de Granada". Documento de Sintesis. Consejería de Política Territorial. J. A. Sevilla.

FEDERATION DES PARCS NATURELS DE FRANCE (1983). "Les Parcs Naturels et le patrimoine ethnologique".

HURTADO GALlARDO, P. (1984). "Exposierra-84". Revista de Estudios Andaluces, n. ${ }^{\circ}$ 3, pp. 166-167.

JUNTA DE ANDALUCIA (1981), "Catalogación y Conservación de Espacios Naturales Protegibles. Documento-Marco". Consejerfa de Política Territorial. J. A. Granada.

MARCHENA GOMEZ, M. (1984). "Espacio, ocio y turismo en Andalucía". Revista de Estudios Andaluces, n.0 2, pp. 129-149.

PAYNo GALBARRIATO, J. A. (1983). "Claves para el desarrollo económico de Andalucía". Junta de Andalucía. Fundación Banco Exterior. Sevilla.

RUIZ DE URRESTARAZU, E. (1983). "Algunos estudios operativos sobre áreas a proteger y de esparcimiento en la Comunidad Autónoma Vasca". Actas VIII Coloquio de Geógrafos Españoles, Barcelona, pp. 220-227.

UICN, PNUMA, WWF. (1980). "Estrategia Mundial para la Conservación". Roma.

VERDE (1984). "Plan Especial de Protección del Medio Físico y Catálogo de Espacios y Bienes Protegibles de la Provincia de Córdoba". Documento de Síntesis. Consejería de Política Territorial. J. A. Sevilla. 